\title{
Microarray analysis for constitutional cytogenetic abnormalities
}

Lisa G. Shaffer, $P h D^{1}$, Arthur L. Beaudet, $M D^{2}$, Arthur R. Brothman, PhD ${ }^{3}$, Betsy Hirsch, $P h D^{4}$, Brynn Levy, PhD , Christa Lese Martin, PhD ${ }^{6}$, James T. Mascarello, $\mathrm{PhD}^{7}$, Kathleen W. Rao, $P h D^{8}$

A Working Group of the Laboratory Quality Assurance Committee of the American College of Medical Genetics

Key Words: array comparative genomic hybridization, microarray, single nucleotide polymorphism, oligonucleotide,

fluorescence in situ hybridization, BAC, copy number

Disclaimer: This guideline is designed primarily as an educational resource for health care providers to help them provide quality medical genetic services. Adherence to this guideline does not necessarily ensure a successful medical outcome. This guideline should not be considered inclusive of all proper procedures and tests or exclusive of other procedures and test that are reasonably directed to obtaining the same results. In determining the propriety of any specific procedure or test, the geneticist should apply his or her own professional judgment to the specific clinical circumstances presented by the individual patient or specimen. It may be prudent, however, to document in the patient's record the rationale for any significant deviation from this guideline.

Conventional cytogenetic analysis allows for the identification of balanced and unbalanced structural and numerical abnormalities. Chromosome analysis may be requested by a physician to confirm a specific diagnosis, such as Down syndrome, but often it is performed to examine the whole genome at the resolution of the metaphase chromosome to look for an unspecified imbalance that accounts for a patient's clinical issues. Thus, conventional cytogenetic analysis is usually not dependent on the patient's phenotype because all chromosomes and all bands at particular resolutions are examined. The diagnostic yield of banded chromosome analysis in children with global developmental delay is approximately $3.7 \% .^{1}$

Molecular cytogenetic techniques, such as fluorescence in situ hybridization (FISH), were introduced into the clinical cytogenetics laboratory to increase resolution. FISH is targeted to a particular chromosomal region or regions. Testing may be locus specific as indicated by the patient's phenotype, or it may be used to interrogate multiple loci, such as the subtelomeric regions. Using

\footnotetext{
From ${ }^{1}$ Signature Genomic Laboratories, Spokane, Washington; ${ }^{2}$ Baylor College of Medicine, Houston, Texas; ${ }^{3}$ University of Utah School of Medicine, Salt Lake City, Utah; ${ }^{4}$ University of Minnesota Medical School, Minneapolis, Minnesota; ${ }^{5}$ College of Physicians and Surgeons of Columbia University, New York, New York; ${ }^{6}$ Emory University School of Medicine, Atlanta, Georgia; ${ }^{7}$ Genzyme Genetics, Santa Fe, New Mexico, ${ }^{8}$ University of North Carolina, Chapel Hill, North Carolina.

Approved by Board of Directors, March 21, 2007. Go to www.geneticsinmedicine.org for a printable copy of this document.

American College of Medical Genetics, 9650 Rockville Pike, Bethesda, MD 20814-3998.

Disclosure: L.G. Shaffer has ownership, receives consulting fees, and sits on the Members' Board of Signature Genomic Laboratories, LLC. A.L. Beaudet is Chair of the Department of Molecular and Human Genetics at Baylor College of Medicine (BCM) which offers extensive genetic laboratory testing, including use of arrays for genomic copy number analysis, and derives revenue from this activity. BCM has a collaborative marketing agreement with Athena Diagnostics and currently uses arrays manufactured by Agilent Technologies. B. Levy is on the Affymetrix scientific adivsory committee for cytogenetics.
}

DOI: $10.1097 /$ GIM.0b013e31814ce3d9 panels of subtelomere probes, the diagnostic yield of clinically relevant rearrangements is approximately $2.5 \%{ }^{2}$

Comparative genomic hybridization (CGH) was developed to aid in the identification of unbalanced rearrangements using a patient's DNA as the probe for the analysis. By comparing the patient's DNA with a reference sample, gains and losses were first identified at the resolution of the metaphase chromosome because the DNA samples were hybridized to normal metaphase chromosomes. ${ }^{3}$ However, due to the limited resolution of the metaphase chromosome, CGH was adapted for use on microarrays. ${ }^{4}$ In array-based CGH, pieces of human genomic DNA (in the form of oligonucleotides, bacterial artificial chromosomes (BACs), or other DNA sequences) are attached to a solid support (e.g., a glass microscope slide) and the patient and reference DNAs are differentially labeled and applied to the microarray. ${ }^{5}$ Single nucleotide polymorphism (SNP) microarrays can also be used for genomic copy number assessment. These arrays differ from CGH arrays in that only the patient's genome is hybridized to the solid support. The intensity of the SNPs indicate DNA copy number gains and losses when compared with a standard set of SNP intensities derived from normal reference controls.

The diagnostic yield of microarray analysis is dependent on the genomic coverage contained on the microarray. Preliminary studies using limited BAC arrays have yielded estimates of approximately $10 \% .6,7$

Microarray analysis allows for the simultaneous analysis of hundreds or thousands of discrete loci, not possible within a single FISH experiment and at a much higher resolution than conventional cytogenetic analysis. As in conventional cytogenetic analysis, microarrays can be used to detect unbalanced regions of the genome. Although current microarray technologies cannot identify balanced rearrangements and some ploidies, ${ }^{8}$ most chromosome analyses that are performed on 
individuals with phenotypic abnormalities, developmental delays, or mental retardation are performed to detect unbalanced chromosomal rearrangements, those resulting in net gains and losses of chromosomes or chromosomal segments. Thus, the use of microarray analysis in the clinical cytogenetics laboratory will not change the approach to the child who has traditionally received a cytogenetic analysis and may replace the "stepwise" approach of multiple FISH studies in patients with unclear etiologies. In addition, microarray analysis can be used as an adjunct to conventional cytogenetics to further clarify chromosome abnormalities detected by G-band analysis, avoiding the need for multiple FISH assays.

As with conventional and molecular cytogenetic studies, chromosome abnormalities of unclear clinical significance are sometimes uncovered by microarray analysis. These unclear results require the cytogenetic analysis of parents or other relatives to fully interpret the abnormal finding. Through the testing of parents or by FISH confirmation studies, many of these genomic alterations can be clarified. Thus, the situations encountered by microarray analysis are not unlike those that were experienced early on in the clinical cytogenetics laboratory in the elucidation of chromosomal heteromorphisms, nor unlike the finding of a novel subtle abnormality by conventional G-banding.

In summary, array-based analysis is a new molecular cytogenetics tool that is used to identify the cytogenetic basis of genetic disease. The results obtained using this new tool are correlated with decades of clinical genetic knowledge and publicly available information from the Human Genome Project. The expertise required for performing and interpreting microarray data are not substantially different from the skill set and experience required for interpretation of karyotypes and FISH that clinical cytogeneticists already possess. Thus, the use of microarray analysis will enhance the patient care already provided by clinical cytogenetic laboratories.

\section{E13 MICROARRAY ANALYSIS FOR CONSTITUTIONAL CYTOGENETIC ABNORMALITIES}

\section{E13.1 General considerations}

Constitutional cytogenetic abnormalities include aneuploidy (extra or missing chromosomes) and structural aberrations (deletions, duplications, translocations, inversions, marker chromosomes). Microarray analysis by CGH-based or SNP-based arrays can detect DNA copy number gains and losses, thereby identifying unbalanced chromosome abnormalities, including unbalanced microscopic and submicroscopic abnormalities.

This technique cannot detect balanced rearrangements, some ploidy changes, and mutations (nucleotide base pair changes). Uniparental disomy cannot be detected by CGHbased arrays but may be uncovered using SNP-based arrays.

Low-level mosaicism for unbalanced rearrangements and aneuploidy may not be detected by array CGH. Each laboratory must determine the sensitivity of the microarray for detection of mosaicism.
Genomic microarrays typically consist of pieces of DNA attached to a solid support (e.g., glass microscope slide). Depending on the design of the array, the extent to which individual clones or probes on the array can be validated, and the clinical indication for testing, an array may be used either as an adjunct to more established testing methods, such as routine chromosome analysis and targeted FISH assays, or as a primary diagnostic tool for detecting chromosomal abnormalities.

The sensitivity of this test depends in part on the number, distribution, and size of the clones/probes on the array and the algorithmic parameters used in the data analysis.

\section{E13.2 Validation}

Various types of microarrays may be used for the diagnosis of chromosome abnormalities in a clinical laboratory: those that are U.S. Food and Drug Administration (FDA) approved and available through a commercial source, IUO (investigational use only) or RUO (research use only) microarrays, which are commercially available and validated within the laboratory that intends to use them as a clinical tool, and those that are "home-brew," developed within the laboratory that intends to use them as a clinical tool. Depending on the intended clinical application of the array, each of the types of arrays may have different levels of validation.

\section{E13.2.1 FDA-approved commercially available microarrays to} be used as adjunctive or as a replacement to established cytogenetics and FISH

[Note: At the time of the publication of these Guidelines, there are no commercially available FDA-approved microarrays for this application. However, laboratories are advised to keep abreast of new developments in this rapidly developing technology.]

For FDA-approved arrays that are used to replace established methodologies (e.g., traditional cytogenetic analysis, FISH), the laboratory should run a series of normal and abnormal controls to establish performance parameters of the array (E13.2.4.1). To the extent possible, the laboratory should use abnormal controls that represent abnormalities that the array is designed to detect.

For FDA-approved arrays that are to be used as adjuncts to traditional cytogenetic analysis and clinically targeted FISH assays, validation may be accomplished by running a series of normal controls and abnormal controls that in combination address the regions of the genome represented on the array.

The method and scope of validation must be documented within the laboratory and the concomitant limitations of the analysis must be described in the laboratory report.

\section{E13.2.2 IUO or RUO commercially available microarrays to be used as adjunctive or as a replacement to established cytogenetics and FISH}

For IUO/RUO arrays that are used to replace established methodologies (e.g., traditional cytogenetic analysis, FISH), the laboratory should aim to validate each clone or region on 
the microarray using at least one reference DNA with a known abnormality for each disorder represented on the array.

For IUO/RUO arrays that are to be used as adjuncts to traditional cytogenetic analysis and clinically targeted FISH assays, validation may be accomplished by running a series of normal controls and abnormal controls that in combination address the regions of the genome represented on the array.

The method and scope of validation must be documented within the laboratory and the concomitant limitations of the analysis must be described in the laboratory report.

\section{E13.2.3 Home-brew microarrays to be used as adjunctive or as a replacement to established cytogenetics and FISH}

For home-brew arrays that are used to replace established methodologies (e.g., traditional cytogenetic analysis or FISH), the laboratory should ideally FISH map each clone to verify cytogenetic map location before constructing the array. Alternatively, the laboratory may provide additional evidence to verify clones through end-sequencing or PCR amplification of genes of interest.

The laboratory should attempt to validate each clone or region on the microarray using at least one reference DNA with a known abnormality for each disorder represented on the array.

The method and scope of validation must be documented within the laboratory and the concomitant limitations of the analysis must be described in the laboratory report.

\section{E13.2.4 Demonstration of expertise and validation of a new technology in the laboratory}

In addition to validation of the various types of microarrays, the laboratory must demonstrate expertise in array performance and analysis.

The following steps must be conducted by the laboratory before offering diagnostic testing with any of the types of microarray (e.g., FDA approved, IUO/RUO, home-brew). This process is necessary whether the laboratory is using microarrays for the first time, changing microarray platforms (e.g., BAC arrays, oligonucleotide arrays), or changing microarray manufacturers.

\section{E13.2.4.1 Validation of new microarrays (new platform or new manufacturer)}

New microarrays to the laboratory should be validated with DNA representing as many chromosomal abnormalities as possible to obtain the experience and confidence needed to perform microarray testing. A minimum of 30 different chromosomally abnormal specimens should be tested.

In addition, the laboratory should exchange five samples (normal or abnormal in a blinded fashion) with another reference laboratory providing microarray testing. After this initial validation period, new microarray lots can be validated as described in E13.2.4.3.

\section{E13.2.4.2 Validation of a new (version of a) microarray established in the laboratory}

This validation does not apply to new platforms from the same manufacturer (see E13.2.4.1). The intent of this validation is to validate an enhanced (new version) of a microarray that has been previously validated for diagnostic use in the laboratory. The laboratory should validate a new microarray (e.g., enhanced or new version) using five abnormal specimens to assess accuracy of performance. If possible, the abnormal specimens should reflect the additional regions of the genome or syndromes on the new microarray under validation.

\section{E13.2.4.3 Validation of a new lot of the same microarray established in the laboratory}

The intent of this validation is to reasonably assess that the new lot performs as the previous lot. The manufacturer should supply documentation of the quality control comparison between lots. In addition, each new lot should have documentation by the manufacturer that printing of each clone was successful (visualization of spots using a DNA-specific stain) for BAC arrays or synthesis of each oligonucleotide was successful (synthesis verification) for oligonucleotide arrays. For new lots, one (preferably) abnormal specimen is repeated on the new lot and compared with the result from the old lot to establish equivalency.

In addition, it is advisable that each new lot of a microarray be validated with two samples from chromosomally normal individuals (these may be the control samples) to identify: problems in the printing (poor spots) of clones or synthesis of oligonucleotides, problems in reagents (poor target DNA quality, suboptimal reagents), or problems in the analysis.

\section{E13.2.5 Proficiency testing}

The laboratory should establish internal proficiency testing of normal and abnormal specimens for microarray analysis as part of the laboratory's internal quality assurance program and ongoing quality improvement program.

In the absence of the availability of an external proficiency program for microarray analysis by a deemed entity (e.g., CAP), the laboratory should establish external proficiency testing of normal and abnormal specimens for microarray analysis as part of its quality assurance program and ongoing quality improvement program. Deidentified normal and abnormal DNA specimens should be exchanged (in a blinded fashion) with another reference laboratory performing microarray testing. Alternatively, DNA specimens could be received from an outside cytogenetics laboratory and correlation between the cytogenetic or FISH results and the microarray results may be sufficient to provide ongoing proficiency. Proficiency testing should include both normal and abnormal specimens and should be performed according to CLIA ' 88 guidelines.

When an external proficiency testing program becomes available through an appropriate deemed organization, the laboratory should be encouraged to enroll and participate in all relevant challenges. 
Documentation of participation and the performance results of all internal and external proficiency tests must be retained by the laboratory and made available to all accreditation agency inspectors.

\section{E13.3 Analytic standards}

Monitoring preanalytical variables, analytical variables, and postanalytical variables should be part of the laboratory's quality assurance and quality improvement programs. Such variables may include quality of the specimen received, number of hybridization failures, and number of cases requiring parental studies.

\section{E13.3.1 Array design}

The performance of microarray analysis in detecting chromosome aberrations is dependent on the resolution of the array, which is limited by the size of the DNA targets and the distance between those sequences naturally located on the chromosome. BAC microarrays typically have cloned DNA targets with an average size of $160 \mathrm{~Kb}$, whereas oligonucleotide probes range in size from 25 mers to 75 mers.

Microarrays may be developed for specific regions of the genome (targeted genome microarrays) for detection of unbalanced subtelomeric rearrangements, aneuploidy, particular microdeletion/microduplication syndromes, or a genomewide array with a specified distribution/spacing of clones/ probes (for example, $1 \mathrm{Mb}$ array; one oligonucleotide/SNP per $300 \mathrm{~kb}$ array), or microarrays may be developed for the entire genome using overlapping (contiguous) clones (whole genome microarrays) or high-density oligonucleotides. The coverage of the various commercial oligonucleotide arrays currently range in size from one probe per $6 \mathrm{~kb}$ to one probe per 70 $\mathrm{kb}$.

The microarray targets may include large insert clones [BACs, P1-derived artificial chromosomes (PACs)], smaller insert clones (cosmids, fosmids, plasmids), or smaller DNA segments (oligonucleotides, cDNA). Yeast artificial chromosomes are generally not recommended because they are often chimeric and would have suboptimal utility in diagnostic applications.

Multiple clones/probes representing any single clinically relevant locus provide additional diagnostic information and should be included on the array. Comparison of the microarray results among these clones/probes at a single locus may provide a more sensitive assay. Contiguous clones/probes spanning a large region provide supplementary diagnostic information regarding size of deletions or duplications as an adjunct to clinical and other laboratory findings. The laboratory should establish the number of clones/probes used to determine an abnormal threshold.

\section{E13.3.1.1 Considerations for BAC array design}

If possible, each clone should be represented multiple times on the microarray (minimum of two to four times) and preferably in more than one location on the microarray to increase test reliability and reduce uninformative or false-positive/- negative results due to technical artifact. The array should contain a set of normalization clones for which a normal ratio is established after each microarray analysis. The laboratory should determine and document the method for normalization. In some instances, this may be provided by the manufacturer.

Clones may be selected from available public databases and obtained through a public or private source. Regardless of whether the microarray is a home-brew or manufactured by a commercial entity, the identity of each clone should be verified through end-sequencing, FISH localization, or some other comparable method. This verification should be documented within the laboratory quality control plan for home-brew arrays or provided to the end user by the manufacturer for commercially manufactured arrays. The laboratory director should make every effort to obtain documentation of clone validation from the manufacturer.

Clones not mapping to the proper/intended locus should be discarded from the array or omitted from the final laboratory analysis.

\section{E13.3.1.2 Considerations for oligonucleotide array design}

Oligonucleotide arrays should be designed with consideration of the statistical algorithms that will subsequently be used for determining abnormal thresholds. The number of probes within a given region of interest (e.g., within a region known to be associated with a clinical syndrome) should be sufficient such that the assay will have the sensitivity necessary for detection of an abnormality. For example, if applying an algorithm that requires 10 oligonucleotides within a $0.3 \mathrm{Mb}$ region to fall outside of the normal ratio as a cutoff, the laboratory director needs to ensure that there is a minimum of 10 oligonucleotides within the critical region to detect a deletion or duplication therein. For oligonucleotide arrays that are not customized for the laboratory, it is essential that the director familiarize himself/herself with the coverage on the array; even arrays that have an "average" spacing of as little as $10 \mathrm{~kb}$ may have significant gaps that are clinically relevant. There should also be redundancy of a subset of probes on the array, with the redundant probe sequences being represented at more than one location on the microarray. The oligonucleotide CGH arrays should contain a set of normalization probes (e.g., negative spots that should not hybridize to human DNA and positive saturated spots) for which a normal ratio is established for each analysis. The laboratory should determine and document the method for normalization. In some instances, this may be provided by the manufacturer. For SNP arrays, a set of control probes should be included on the array for the purpose of determining a threshold for abnormal results.

Probes selected from the public domain should be listed with their physical and cytogenetic positions on the human genome. All probe descriptions and annotations should be openly accessible. Details regarding design and synthesis verification as well as all quality control steps taken to validate and assess the performance/reproducibility of the array should be documented and provided by the manufacturer. 


\section{E13.3.2 Specimen requirements}

\section{E13.3.2.1 Tissue requirements}

Microarray analysis may be performed after DNA extraction of any specimen that yields DNA (e.g., peripheral blood, cord blood, skin fibroblasts, fixed-cell pellet, paraffin-embedded tissues). The laboratory should establish the specimen requirements for microarray analysis for each tissue type. However, as the signal-to-noise ratio will likely differ for each of these specimen types, the laboratory will need to determine normal and abnormal threshold values for each sample type, recognizing that the sensitivity of the assay may differ for each.

The laboratory needs to establish the types of specimens appropriate for array testing. Minimum required quantities of various tissue types, anticoagulants for peripheral blood specimens, and DNA submission requirements must be established by each laboratory.

\section{E13.3.2.2 DNA requirements and processing}

The laboratory should establish the minimum DNA requirements to perform the test. Considerations include whether the test is performed once per specimen or, for array CGH, performed twice in a "dye-swap" or "dye-reversal" strategy.

The laboratory should have written procedures in the laboratory procedure manual and/or quality management program for DNA extraction and labeling, DNA quantification (e.g., fluorometer, spectrophotometer), obtaining adequate quality and concentration of DNA (e.g., examination by gel electrophoresis), proper fragmentation (e.g., via sonication or digestion), and adequate fluorescent labeling (e.g., examination by gel electrophoresis, visual inspection, ultraviolet/visible spectroscopy).

The laboratory should have documentation of these parameters in each patient record.

\section{E13.3.2.3 Suboptimal samples}

If a sample does not meet requirements of the laboratory and is deemed suboptimal, the recommended action is to reject the specimen and request a repeat. If obtaining a repeat specimen is not possible, whole genome amplification could be considered if the laboratory is experienced in this technique and if the potential biases inherent in the technique are detailed in the report so that the physician and patient are informed of the limitations of this technique. Written standards describing when and how the whole genome amplification procedure is performed should be incorporated into the laboratory manual.

\section{E13.3.3 Controls}

\section{E13.3.3.1 Documentation}

Array CGH requires the comparison of a patient result to a control (reference) result and SNP oligonucleotide microarray analysis requires the comparison of a patient result with a set of established normal controls (references). The laboratory should have a written policy concerning the type(s) of control specimens that will be used for each patient specimen type and clinical situation.

\section{E13.3.3.2 Establishing controls}

It is recommended that the laboratory establish both male and female control samples and guidelines that detail whether they are to be used in mismatched (opposite sex) or same-sex comparisons.

The laboratory should establish whether single male and single female specimens will serve as controls or whether combined pools of multiple male and/or multiple female DNAs will be used as controls. The limitations of both approaches should be understood by the laboratory and taken into consideration in the interpretation of results.

In most instances, controls with presumably normal karyotypes are used. However, there may be instances when use of a control with a particular abnormal karyotype might be appropriate. ${ }^{8}$

\section{E13.3.3.3 Validating new lots of controls}

Each new control DNA or new lot of prepared control DNA should be compared by microarray analysis to the previous lot of control DNA.

\section{E13.3.3.4 Controls for specific clinical situations}

The laboratory must establish whether the same controls will be used for all hybridizations or whether different controls will be used for each hybridization. At this time, some laboratories prefer to use individual male and female controls, and some prefer to use pooled male and pooled female DNA as controls. Some laboratories prefer same-sex controls, and some prefer opposite-sex controls for microarray hybridizations. Any of the above strategies would be acceptable. The laboratory should document the specific rationale for choosing certain strategies and have provisions to accommodate the various disadvantages for one strategy versus another for certain situations. Considerations include CNVs carried by the individuals used as controls and whether opposite-sex or same-sex controls are used. Opposite-sex controls provide a built-in "abnormal" result, which may be helpful in assessing the quality of the microarray results through assessment of dose differences between the X and Y chromosomes. Same-sex controls may offer better sensitivity for detecting gains or losses of regions on the $\mathrm{X}$ and $\mathrm{Y}$ chromosomes. However, for some suspected sex chromosome and ploidy abnormalities that may be difficult to distinguish by microarray analysis, the laboratory may choose to perform both same-sex and opposite-sex control hybridizations or may choose to confirm the results by FISH or cytogenetic analysis.

\section{E13.3.4 Microarray data analysis}

\section{E13.3.4.1 BAC arrays}

The laboratory should apply statistical methods to assess the ratio of the control DNA specimen to the patient DNA specimen for each clone. When the clones are spotted more than 
once on the array, the ratios of the control DNA specimen to the patient DNA specimen can be averaged across identical spots. The statistical methods used should be described in the laboratory's procedure manual.

Normalization should be used to establish the baseline ratio of control DNA specimen to the patient DNA specimen before the analysis of the microarray.

The laboratory must establish the normal and abnormal reference ranges (nullisomy, single-copy loss, single-copy gain, and amplification) for each region of the genome represented on the microarray using normal control DNA specimens for validation. The abnormal ranges should be tested using representative known DNA from chromosomally abnormal individuals or cell lines that carry aberrations for gains and losses anticipated in the construction of each particular microarray.

The laboratory must establish methods to present the data obtained from the ratios of the control DNA specimen and the patient DNA specimen. Array data are often depicted as numerical values or graphically represented.

Low-level mosaicism for unbalanced rearrangements and aneuploidy may not be detected by array CGH. Thus, each laboratory should either determine the sensitivity of the microarray for detection of mosaicism or should identify this limitation in the patient report.

\section{E13.3.4.2 SNP arrays}

The laboratory should apply statistical methods to assess the likelihood that a given region of SNP oligonucleotide probes is gained or lost. Signal intensity values from replicate probes may be combined and reported as a single value for each probe sequence. Various algorithms and software exist for determining DNA copy number changes, and a single algorithm/software package should consistently be used for all microarray validations. When switching to an alternate algorithm/software package, reanalysis of the data used for initial validation should be performed to demonstrate comparable performance. Normalization of the data should be considered as it may potentially eliminate technical biases attributable to factors such as differences in scanners, operators, and replicates.

The laboratory must establish the normal and abnormal values that indicate the copy number state (nullisomy, single copy loss, single copy gain, and amplification) for each probe/region of the genome represented on the microarray using normal control DNA specimens for validation. The laboratory should establish the number of clones/probes used to establish a result and determine an abnormal threshold. The abnormal values should be tested using representative known DNA from chromosomally abnormal individuals or cell lines that carry aberrations for gains and losses anticipated based on the design and construction of each particular microarray.

The laboratory must establish methods to present the data obtained from the comparison of the intensity values of the normal control reference set and the patient DNA specimen. Array data are often depicted as numerical values or graphically represented.
Low-level mosaicism for unbalanced rearrangements and aneuploidy may not be detected by microarray analysis. Thus, each laboratory should either determine the sensitivity of the microarray for detection of mosaicism or should identify this limitation in the patient report.

\section{E13.3.4.3 Oligonucleotide arrays}

The laboratory should apply statistical methods to assess the ratio of the control DNA specimen(s) to the patient DNA specimen for each oligonucleotide/probe. The laboratory director must be familiar with the principles of the software program being applied. Specifically, the director must know how an outlying value on one oligonucleotide/probe affects the ratio generated for a neighboring oligonucleotide/probe. Critical to the analysis will be the rule (sometimes called "filters") that are used in the analysis to determine that there is a significant gain or loss of a chromosomal region. Some manufacturers provide standard rules or filters to set the cutoff points. However, the laboratory should rigorously test the rules or filters as part of the validation of the assay. For example, one standard filter that might be used as a criterion for an abnormal result could be 10 oligonucleotides within a $0.5-\mathrm{Mb}$ region with a minimum absolute ratio value (based on a $\log 2$ ratio) of 0.3 . Depending on the density of the array across each region, that rule may be too stringent or too lenient. Thus, it may be necessary to adjust depending on the region being analyzed. Importantly, the moving average across each chromosome should be carefully examined. This is particularly true for cases that are mosaic, which will typically generate absolute ratio values less than that expected for a nonmosaic case and thus may fall below the standard cutoff value. However, such mosaicism may be apparent upon examination of the running averages. Each laboratory should determine the sensitivity of the microarray for detection of mosaicism or should identify this limitation in the patient report.

\section{E13.3.5 Interpretation: copy number variants (CNVs)}

The laboratory director and staff should be familiar with benign CNVs or CNVs of unknown clinical significance on the array as well as the expanding current literature and databases on the more commonly reported CNVs and should interpret the microarray analysis in this context. The laboratory director should also consider the possibility that a clinically significant abnormality can occur in known variant regions. These may include deletions and duplications encompassing a known $\mathrm{CNV}$ or may represent a homozygous alteration of a CNV that would otherwise be benign when heterozygous. CNV databases have been established to assist the laboratory director in making decisions about gains and losses detected by microarrays. Examples of such databases are the Database of Genomic Variants (http://projects.tcag.ca/variation/) and DECIPHER (http://www.sanger.ac.uk/PostGenomics/decipher/).

The laboratory must have a written procedure to determine whether any regions covered by clones or probes on the array represent known regions of benign CNVs within the genome. This may include documentation of established polymorphic 
databases from the laboratory and/or other institutions. It is important that, with the exception of purported benign CNVs, all regions showing abnormal copy number findings be characterized by either FISH, parental studies, quantitative polymerase chain reaction (PCR), or one of the methods noted above, and the laboratory director be familiar with the rapidly changing literature on the location and frequency of these CNV sites.

\section{E13.3.6 Confirmation of abnormal microarray results}

\section{E13.3.6.1 Clone identity}

A justified concern about microarray analysis is confirmation that each spot represents the correct clone. It is nearly impossible to validate each clone once the array is printed. Validation can be achieved if the laboratory has DNA from samples with abnormalities with copy number changes for each clone or locus (region) represented on the microarray. The laboratory director and staff should be acutely aware of any discrepancies in array data potentially attributable to incorrect identification of a clone on a microarray. Discrepancies can be addressed through FISH with the clone in question to metaphase cells of the patient showing the potentially anomalous result.

\section{E13.3.6.2 Probe identity}

Oligonucleotide probes are synthesized by using information from the public domain. The loci used need to be well documented by Human Gene Mapping Workshop, Geneatlas, the Genome Data Base, or publication in the peer-reviewed scientific literature. Given that many thousands of probes are printed on the array, it is virtually impossible to validate each probe once the array is printed. The manufacturer should have vigorous protocols for verifying that synthesis was successful. Although very unlikely, the laboratory director and staff should be acutely aware of any discrepancies in array data potentially attributable to incorrect physical assignment of a probe sequence on the human genome and thus on the microarray. Because current analysis strategies typically consider more than one oligonucleotide for determination of true gains and losses, the effects of an incorrectly assigned oligonucleotide may be negligible. However, should a discrepancy become apparent, the oligonucleotide in question should be excluded from the analysis.

\section{E13.3.6.3 Confirmation of abnormal or ambiguous results}

The laboratory should establish a protocol that allows for confirmation of abnormal or ambiguous microarray results. This may include cytogenetic analysis using banding techniques, FISH, PCR, or other comparable methods.

\section{E13.3.6.3.1 BAC arrays}

Most cytogenetic laboratories may find the use of FISH, with the abnormal clones identified from microarray analysis, as the method of choice for confirmation. The laboratory director must ensure that confirmation can be established and, if con- firmation is to be performed by FISH, that clones are available to the laboratory in a timely manner to maintain clinically relevant turnaround times. The laboratory should have a written policy in place that deals with discrepant results, i.e., when an array shows an abnormality that cannot be confirmed by FISH (the sensitivities of these two tests may differ).

BACs (or other microarray targets) used to confirm microarray results should be validated in the same way that comparable FISH probes or other DNA reagents are validated for clinical use. See E9.2.1.1 of these Standards and Guidelines. This validation can be conducted simultaneously with analysis of the patient's metaphase or interphase cells.

The director may choose to use a probe for confirmation analysis that has been previously validated in the laboratory, if available for the particular region on the microarray showing the abnormality.

During the FISH confirmation of an abnormal microarray result, a control probe may be used in another region on the same chromosome involved in the abnormality. However, if a control probe is not used, another method for the unequivocal identification of the chromosomes may be used (e.g., inverted 4',6-diamidino-2-phenylindole images).

\section{E13.3.6.3.2 Oligonucleotide arrays}

Most cytogenetic laboratories may find the use of FISH as the method of choice for confirmation of abnormal regions. Clones that span the region to be confirmed can be chosen by using publicly available databases. The laboratory director must ensure that confirmation can be established and, if confirmation is to be performed by FISH, that clones are available to the laboratory in a timely manner to maintain clinically relevant turnaround times. The laboratory should have a written policy in place that deals with discrepant results, i.e., when an array shows an abnormality that cannot be confirmed by FISH (the sensitivities of these two tests may differ).

BACs (or other microarray targets) used to confirm microarray results must be validated in the same way that comparable FISH probes are validated for clinical use. See E9.2.1.1 of these Standards and Guidelines. This validation can be conducted simultaneously with analysis of the patient's metaphase or interphase cells.

The director may choose to use a probe for confirmation analysis that has been previously validated in the laboratory, if available for the particular region on the microarray showing the abnormality.

During the FISH confirmation of an abnormal microarray result, a control probe may be used at another region on the same chromosome involved in the abnormality. However, if a control probe is not used, another method for the unequivocal identification of the chromosomes may be used (e.g., inverted 4',6-diamidino-2-phenylindole images).

Alternate molecular methods such as quantitative PCR and multiplex ligation-dependent probe amplification may be used for confirmation, and the use of these techniques is also subject to clinical validation (See G5). 


\section{E13.3.6.4 Parental/family studies}

Parental studies or studies of other family members may be indicated after the identification of some chromosome abnormalities. The laboratory director must evaluate the microarray analysis results and confirmatory test results and determine the best method for parental studies (chromosome analysis, FISH, or microarray analysis). See E13.4.2.4 for general recommendations about when parental studies should be performed.

It will be critical to have relevant clinical data on the patient and family members for interpretation of these follow-up studies.

\section{E13.4 Ordering and reporting}

\section{E13.4.1 Test ordering}

It is at the discretion of the clinician in consultation with the laboratory director as to whether microarray analysis is appropriate for any particular patient.

Whether any specific array should be used as a primary diagnostic tool or as an adjunct to other types of testing including traditional cytogenetic analysis, FISH, or molecular analysis is dependent on the extent to which the loci on the array have been validated and the clinical situation. When the clinical suspicion is high that a patient has a disorder that is presumably diagnosable by the array, and that locus on the array has not been validated, and an established clinical assay is readily available, the array should not be used as a primary diagnostic tool.

The laboratory director is responsible for describing both the advantages and limitations of testing with any particular array so that the clinician can make an informed decision as to the applicability of the array for specific diagnoses.

\section{E13.4.2 Reporting standards}

\section{E13.4.2.1 Turnaround times}

The laboratory should have written standards for microarray analysis test prioritization and turnaround times that are based on the indication for referral (such as newborn with congenital anomalies, child with mental retardation).

These turnaround times should be clinically appropriate.

\section{E13.4.2.2 Clinical relevance of results}

All results should, to the extent possible, be interpreted in the context of other clinical and laboratory findings.

\section{E13.4.2.3 Limitations of testing}

Current microarray analysis technologies will detect only gains and losses of chromosomal segments. Thus, a normal microarray result does not exclude mutations (nucleotide base pair changes) in any gene represented on the microarray and does not exclude a balanced rearrangement or epigenetic events (e.g., uniparental disomy or imprinting mutations).

Additional testing may be appropriate for certain syndromes or conditions when the microarray analysis yields normal results.

\section{E13.4.2.4 Follow-up studies}

FISH or microarray studies of parents or other family members should be recommended when an abnormality is identified by microarray analysis. In some cases, such follow-up testing is performed to exclude a balanced parental rearrangement or the possibility that a parent has a deletion or duplication. In other cases, parental testing is needed when gains or losses are detected by microarray analysis in regions of the genome with unclear clinical significance. In these cases, parental studies may be indicated to distinguish between a familial variant/ population polymorphism and a de novo, possibly clinically relevant alteration.

\section{E13.4.2.5 Reporting microarray analysis results}

The laboratory must determine normal and abnormal ranges for reporting microarray analysis results. The report should include a description of these ranges or the criteria that have been applied to determine abnormal versus normal findings.

The laboratory director should express the microarray results in a meaningful manner using International System for Human Cytogenetics Nomenclature-approved nomenclature (ISCN 2005) ${ }^{9}$ when possible, including any FISH and karyotype results. The microarray results may be expressed using clone designations or in terms of the nucleotide boundaries for any gains or losses in copy number or may list genes partially or completely within the altered region. If this format is used, a specific genome browser nucleotide numbering must be specified (e.g., March 2006 assembly) in the report interpretation, and some indication of the breakpoints should be given to the extent that the technology permits. Whether International System for Human Cytogenetics Nomenclature or nucleotide positions or both are used to specify results, a written interpretation must be provided explaining the findings in a manner understandable to the average health care professional. Results of confirmation studies should also be reported.

Reporting of any questionable variant regions should include a comment noting that CNVs exist, and interpretation of clinical significance should be made with caution.

The laboratory report should provide recommendations regarding parental/family studies including the optimal method of testing for relatives and that genetic counseling should be sought.

If the array is of the variety that can only be applied as an adjunct to, but not a replacement for, traditional cytogenetic analysis and targeted FISH assays, this limitation of use must be clearly stated on the final laboratory report.

Technical information about the microarray being used (e.g., commercial source, number and types of clones and loci represented, and lot number) should be included on the report.

Additionally, the report should provide the limitations of the test in a disclaimer. An example disclaimer is as follows:

This microarray was constructed by (insert name of company) for the sole purpose of identifying DNA copy number gains and losses associated with chromosomal imbalances. This microarray will detect (list detection capabilities, which 


\section{Shaffer et al.}

may include aneuploidy, deletions, and duplications) of the loci represented on the microarray. It will not detect balanced alterations (reciprocal translocations, Robertsonian translocations, inversions, and balanced insertions), point mutations, or imbalances of regions not represented on the microarray and may not detect low levels of mosaicism. The failure to detect an alteration at any locus does not exclude the diagnosis of any of the disorders represented on the microarray. This test was developed and its performance characteristics determined by (your laboratory name here) as required by CLIA ' 88 regulations. It (has/has not) been cleared or approved for specific uses by the U.S. Food and Drug Administration. Pursuant to the requirements of CLIA ' 88 , this laboratory has established and verified the test's accuracy and precision.

\section{References}

1. Shevell M, Ashwal S, Donley D, Flint J, et al. Practice parameter: evaluation of the child with global developmental delay: report of the Quality Standards Subcommittee of the American Academy of Neurology and the Practice Committee of the Child Neurology Society. Neurology 2003;60:367-380.

2. Ravnan JB, Tepperberg JH, Papenhausen P, Lamb AN, et al. Subtelomere FISH analysis of 11,688 cases: an evaluation of the frequency and pattern of subtelomere rearrangements in individuals with developmental disabilities. J Med Genet 2006;43:478489.

3. Kallioniemi OP, Kallioniemi A, Sudar D, Rutovitz D, et al. Comparative genomic hybridization: a rapid new method for detecting and mapping DNA amplification in tumors. Semin Cancer Biol 1993;4:41-46.

4. Solinas-Toldo S, Lampel S, Stilgenbauer S, Nickolenko J, et al. Matrix-based comparative genomic hybridization: biochips to screen for genomic imbalances. Genes Chromosomes Cancer 1997;20:399-407.

5. Albertson DG, Pinkel D. Genomic microarrays in human genetic disease and cancer. Hum Mol Genet 2003;12:R145-152.

6. Shaffer LG, Bui T-H. Molecular Cytogenetic and Rapid Aneuploidy Detection Methods in Prenatal Diagnosis. Am J Med Genet (in press).

7. Stankiewicz P, Beaudet AL. Use of array CGH in the evaluation of dysmorphology, malformations, developmental delay, and idiopathic mental retardation. Curr Opin Genet Dev 2007;17:182-192.

8. Ballif BC, Kashork CD, Saleki R, Rorem E, et al. Detecting sex chromosome anomalies and common triploidies in products of conception by array-based comparative genomic hybridization. Prenat Diagn 2006;26:333-339.

9. Shaffer LG, Tommerup N, editors. ISCN 2005. Basel, Switzerland: Karger, 2005. 